\title{
Facet tracking based driver fatigue monitoring and warning system
}

\author{
S Janardhanarao ${ }^{1}$, R Shankar $^{2}$, Syed Inthiyaz ${ }^{3}$, Syed Shameem ${ }^{4}$ \\ Professor $^{1}$, Associate Professor ${ }^{2,3,4}$ \\ ${ }^{1,2}$ Department of CSE, ${ }^{3,4}$ Department of ECE, Koneru Lakshmaiah Education Foundation, Vaddeswaram, \\ Guntur, India
}

\begin{abstract}
The main idea behind this project is to develop a nonintrusive system which can detect fatigue of the driver and issue a timely warning. Since a large number of road accidents occur due to the driver drowsiness, this system will be helpful in preventing many accidents, and consequently save money and reduce personal suffering. This system will monitor the driver's eyes using camera and we can develop an algorithm with which we can detect symptoms of driver fatigue early enough to avoid accident. [1] So this project will be helpful in detecting driver fatigue in advance and will give warning output in form of sound. Moreover, the warning will be deactivated manually rather than automatically. So, for this purpose a deactivation switch will be used to deactivate warning. [2] Moreover if driver felt drowsy there is much more possibility of accidents to occur. When the driver enters the cabin, first he/she is detected for alcohol consumption, face detection and seat belt detection. [3] If all three conditions get satisfied, then only the vehicle gets started. [4] So, when the driver feels drowsy, a warning buzzer will be produced and also the speed of the vehicle can be controlled by connecting the output of microcontroller (AT89S52) to engine of the vehicle.
\end{abstract}

Key words: Face detection, Fatigue monitoring, Warning system and Speed control system.

\section{INTRODUCTION}

Face tracking-based driver fatigue and monitoring system is ability to investigate the state of the driver. In this project, driver fatigue is detected with the assistance of MATLAB based face monitoring, alcohol consumption and seat belt sensors which are connected to the microcontroller. If any of these conditions are not satisfied, the vehicle will not get started.

There are several technologies for fatigue detection and can be separated as: behavioral parameter based, vehicular parameter based, physiological parameter based. [5] The first method measures eye width, face parameter detections, yawning, head postures. This method requires very accurate measurements. The second method measures movements of the vehicle like steering wheel movement, lane detection, steering wheel grip force etc.., The main goal of these techniques is to observe driving patterns and detect a decline in driving performance due to fatigue and tiredness. [6].

The third method measures physical circumstances such as heart rate, breathing rate, pulse rate, body temperature and respiratory rate etc. These biological parameters are more consistent and precise in drowsiness recognition as they are concerned with driver physically what is happening. This method requires through physical interaction with the driver and many types of sensors.

This paper deals with measurement of face parameters such as position of the face, head postures etc.., We will be giving a certain boundaries for head movements out of which if the driver head comes out, then the warning buzzer will be given immediately to avoid the accident by alerting the driver and the speed of the vehicle will also be reduced at the same time to decrease the extent of accident (if happened).

\section{METHODOLOGY}

\subsection{Face-detection Model:}

Human face localization and detection is often the first step in applications such as video surveillance, Human computer interface, and face recognition. [7] To interface computer with human face, we first access the camera through matlab and then detect the face using vision.CascadeObjectDetector algorithm. We also create boundaries using the keyword Maxsize. By varying the parameters in the maxsize, we can change the size of the frame. Then the face should be detected within the frame. The process of face detection is continuous i.e., it will be monitoring the face of the driver continuously. If the driver face is not detected before the vehicle is started in the given format, [8] the vehicle will not get started. If the driver face moves away from the frame when the vehicle is in motion, then the changes will be detected and the warning buzzer is given.

The output from the computer cannot be given directly to the controller to give a warning buzzer if any change is identified like driver face goes out of frame. Hence, we use an IC Max 232 which converts the high voltage output from the computer to low voltage levels of controller.

The system is designed to minimize the road accidents which is caused by driver faults. The faults of driver may 
happen during the long journey The continuous driving system or sleepless nights may bring him to get drowsiness. The drowsiness causes the road accidents. The proposed system is developed to avoid such things like by giving caution alarm and by giving vibration sensation to body. [9]

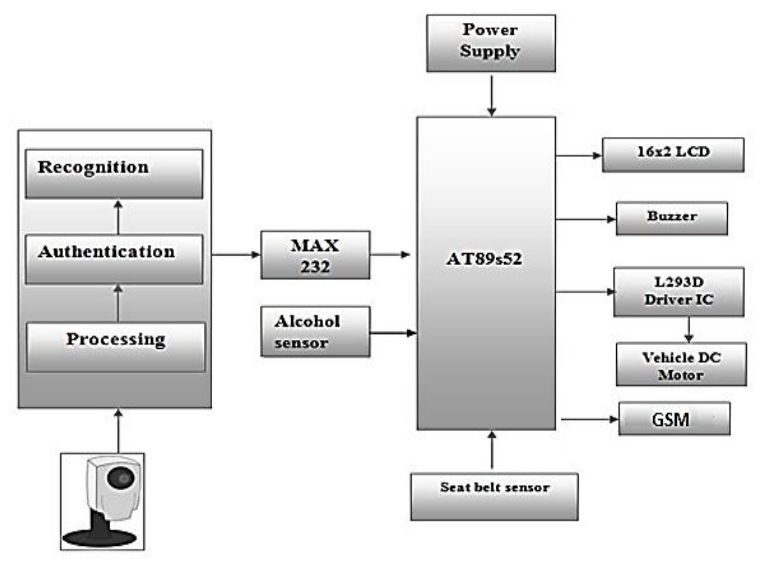

Figure 1: Block diagram

The arrangement uses the microcontroller which is interfaced with alcohol sensor, seat belt sensor and PC loaded with MATLAB, DC motors, Buzzer alarm. The alcohol module digital output is given to P1.1 and seat belt sensor such as SPDT limiting switch is interfaced with P1.3, LCD interfaced with port $2(\mathrm{P} 2), \mathrm{PC}$ is interfaced with serial port (P3.0, P3.1) through a level converter such as $\max 232$, the buzzer is interfaced with P3.7, vehicle Engine motor is given to P1.6 \& P1.7 through a driver IC called as L293D.

When system starts, the microcontroller checks all the input signals from input devices. The web camera of PC gets opened by MATLAB simulation and the camera starts capturing the face images. If the face is in the same frame, then the microcontroller gets an active high signal. Simultaneously microcontroller reads the data from alcohol sensor and seat belt sensor.[10],[11],[12],[13][14],[15] If the seat belt is not wearing, the microcontroller reads an active low signal. Then MCU gives active low signal to motor to get OFF the engine and buzzer get active low signal which activates buzzer to give alarm. When microcontroller reads an active high signal from seat belt sensor, the motor does not ON until all other authentications are successful. If alcohol is consumed, the sensor gives active low signal through P1.1 and MCU give active low signal to motor to get OFF and buzzer to get ON. Face recognition data is transmitted from PC through serial communication. If face is not detected character ' 0 ' is sent to $\mathrm{MCU}$ as 8 bits (10bit Frame) and Motor get OFF and buzzer get ON.

If alcohol sensor detects no consumption alcohol, it gives active high signal and if seat belt wears, it gives active high signal and if serial port receives character ' 1 ' ( 8 bits) by face recognition, the motor / engine starts and buzzer get OFF.

\section{RESULTS AND DISCUSSION}

In this section, we present the experimental results obtained in face tracking-based driver fatigue monitoring and warning system. The experiments are conducted in matlab version R2016a and above in windows 10 operating system on computer with Intel core i5 processor $2.30 \mathrm{GHz}$ with $4 \mathrm{~GB}$ RAM. The sample videos are captured and sent to the microcontroller unit through $\max 232$ and all the sensors are verified like alcohol sensor and seat belt sensor. Then the operation is verified. when all conditions are satisfied, When face is not detected and buzzer is ON (shown with green LED)

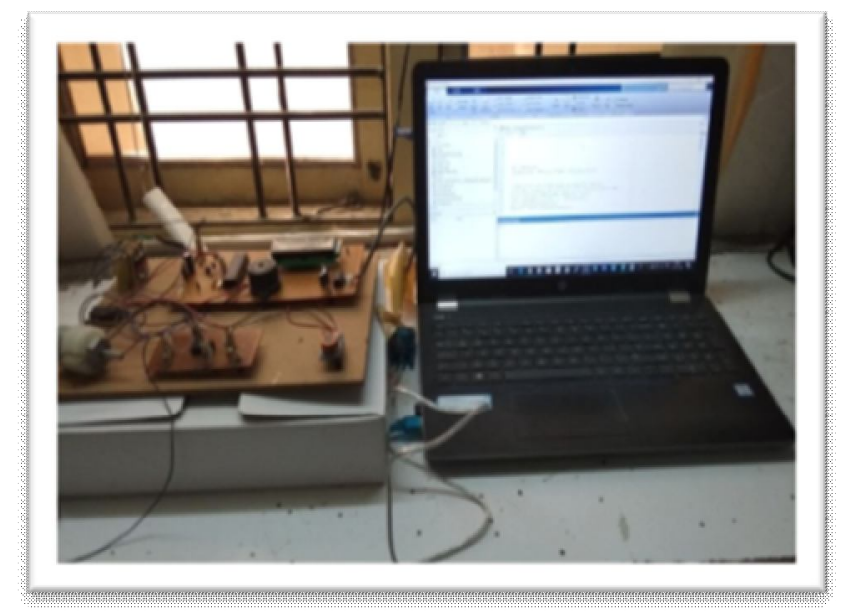

Figure 2: Hardware setup

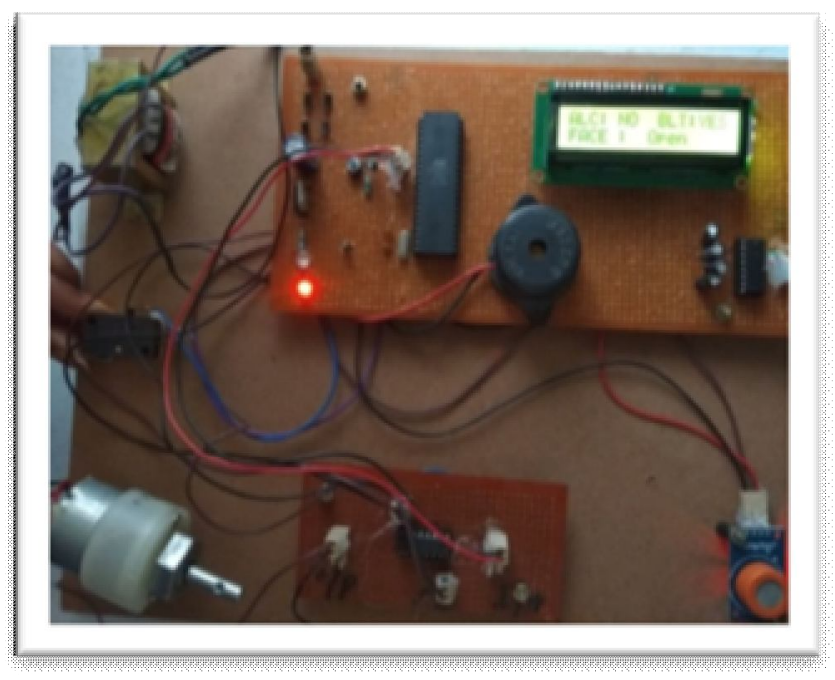

Figure 3: Face recognition output 


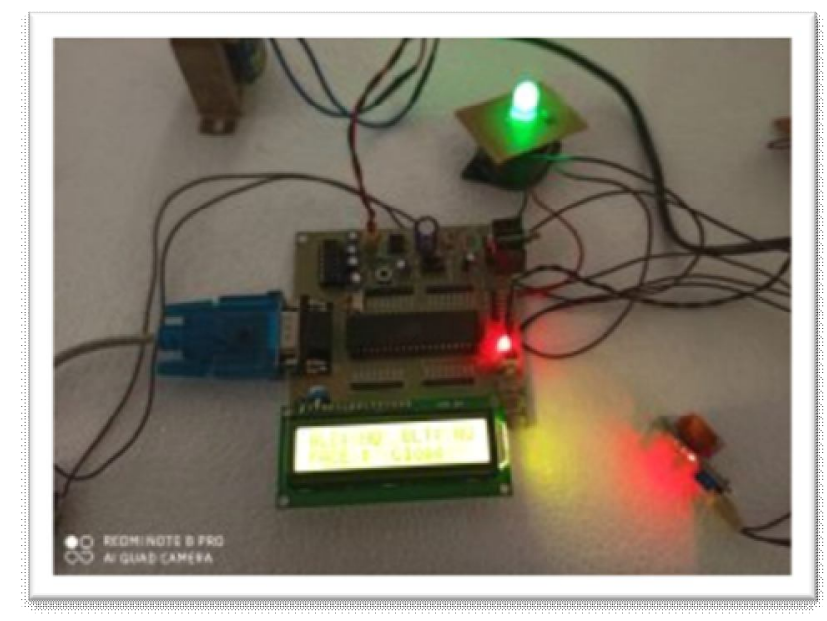

Figure 4: Seat Belt output result

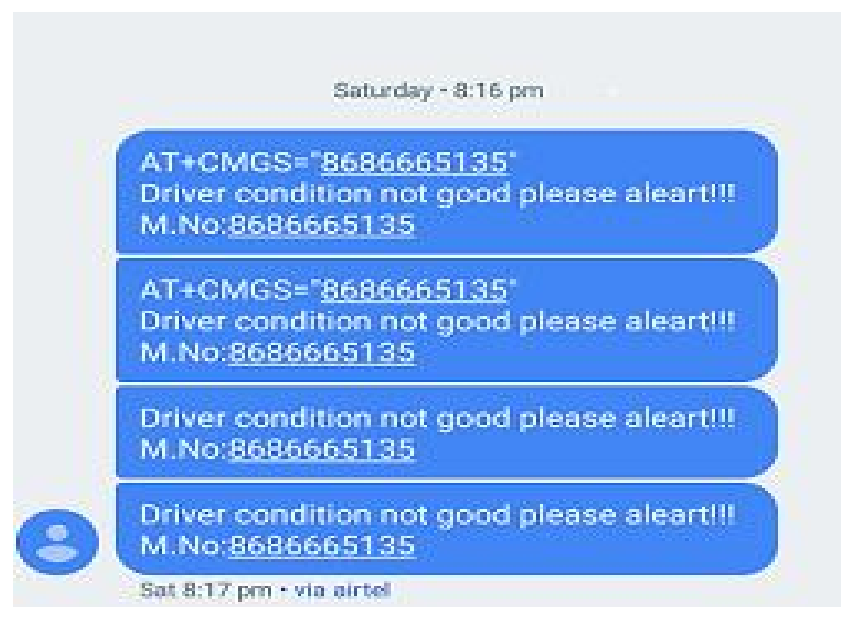

Figure 5: SMS through GPS

An SMS alert is sent to the management or the user who can respond to the condition of the driver. This is done by the data sent from the GSM, GSM sends an alarming message to the mobile numbers stored in it with the condition of the driver. Thus the user can respond in case of emergency and alert hospitals and police. By this the accidents can be avoided and lives of driver and passenger can be saved

\section{CONCLUSION}

At this point in time, a lot of research efforts have gone towards developing a robust driver fatigue detection system. However, results thus far have shown that every fatigue detection technique has some drawback, mainly due to the variability among human behavior. It is therefore necessary to combine different and possible unrelated metrics of fatigue to achieve the best results. Many researchers have studied fatigue detection. Even though a significant number of publications can be found in this field, fundamental problems still need to be solved. The false finding rate of drowsiness is still high in most of the researches. It is at the rate of $10 \%$ at minimum as far as we know according to our literature survey. The outcome of most of the systems is an audible alarm. [16],[17],[18]

Our future plan is to design a driver safety assistant system using in vehicle Video camera. It is a real-time recognition system which uses vision sensors to detect Passengers and driver fatigue conditions. The system assesses the ability of conducting safe driving and notifies the driver for any dangerous situation. Because one of the bottleneck challenges of object recognition is finding efficient and discriminative descriptors that are invariant even in difficult illumination cases. And since the observation of human visual perception shows it is well-adapted to extracting local structural visual information. We plan to propose a new method to mimic the human vision system for artificial fatigue recognition system. Moreover, safety actions are to be performed by an embedded vehicle controlling system to give more efficiency for the system. [19-23]

\section{REFERENCES:}

1. S. Lal, A. Craig, P. Boord, L. Kirkup, and H. Nguyen, "Development of an algorithm for an eeg-based driver fatigue countermeasure," Journal of Safety Research, vol. 1, no. 34, pp. 321-328, February 2003.

2. B. Jap, S. Lal, P. Fischer, and E. Bekiaris, "Using eeg spectral components to assess algorithms for detecting fatigue," Expert Systems with Applications, vol. 36, no. 2, pp. 2352-2359, March 2009.

3. Horng, W.B.; Chen, C.Y. \&amp; Chang, Y.(2004). Driver Fatigue Detection Based On Eye Tracking and Dynamic Template Matching, Proceeding of the 2004 IEEE International Conference on Networking, Sensing \&amp; Control, pp.7-12,ISBN:0-7803-8193-9, Taipei, Taiwan, March 21-23,2004.

4. B. Cheng, W. Zhang, Y. Lin, R. Feng, X. Zhang, "Driver drowsiness detection based on multisource Information," Hum. Factors Ergon. Manuf. Serv. Indust. 22, pp. 450-467, 2012

5. Y. Guosheng, L. Yingzi, B. Prabir, "A driver fatigue recognition model based on information fusion and dynamic Bayesian network," Inform. Sci., 180, pp. 1942-1954, 2010.

6. P. Viola and M. Jones, "Rapid Object Detection using a Boosted Cascade of Simple Features", in Proc. of the IEEE Conference on Computer Vision and Pattern Recognition (CVPR), pp. 511-518, 2001.

7. Ravikanth, B., Akram, P. S., Ashlesha, V., \& Ramana, T. V. (2017). Tuning operating frequency of antenna by using metasurfaces. Paper presented at the International Conference on Signal Processing, Communication, Power and Embedded System, SCOPES 2016 Proceedings, 2064-2068. doi:10.1109/SCOPES.2016.7955811

8. Durga Indira, N., \& Venu Gopala Rao, M. (2018). Automatic facial expression detection system using single face classifier. International Journal of 
Engineering and Technology(UAE), 7(3.12 Special Issue 12), 1144-1148.

9. Vinothkanna, R., \& Vijayakumar, T. (2019). Using contourlet transform based RBFN classifier for face detection and recognition doi:10.1007/978-3-030-00665-5_176

10. Satyanarayana, P., Jaya Devi, N., Sri Hasitha, S. K., \& Sesha Sai, M. (2018). An enhanced viola-jones face detection method with skin mapping \& segmentation.

11. Vamsi Krishna, M., Bhargav Reddy, A., \& Sandeep, V. (2018). Facial recognition enabled smart door unlock system. International Journal of Engineering and technology

12. RamaKrishna, B., Nagabhushana Rao, M., \& Pittala, R. B. (2018). An algorithm to find the geo-map using the social media-facebook. Journal of Advanced Research in Dynamical and Control Systems, 10(7 Special Issue), 1538-1546.

13. Vinothkanna, R., \& Vijayakumar, T. (2019). Using contourlet transform based RBFN classifier for face detection and recognition doi:10.1007/978-3-030-00665-5_176

14. Naga Lakshmi Prasanna, K., Murali Krishna, B., Sadiya Shireen, S. K., \& Poorna Chander Reddy, A. (2019). FPGA based convolutional encoder for GSM-900

15. Srinivasa Rao, Y., \& Ali Hussain, M. (2019). Adaptive quality of service medium access control protocol for IEEE 802.11 based mobile ad hoc network. International Journal of Innovative Technology and Exploring Engineering, 8(4), 430-433.

16. Inthiyaz, S., Prasad, M.V.D., Usha Sri Lakshmi, R., Sri Sai, N.T.B., Kumar, P.P., Ahammad, S.H., "Agriculture based plant leaf health assessment tool: A deep learning perspective", International Journal of Emerging Trends in Engineering Research 7(11), pp. 690-694.

17. Myla, S., Marella, S.T., Goud, A.S., Ahammad, S.H., Kumar, G.N.S., Inthiyaz, S.," Design decision taking system for student career selection for accurate academic system', International Journal of Scientific and Technology Research 8(9), pp. 2199-2206

18. Prasad, M.V.D., Inthiyaz, S., Teja Kiran Kumar, M., Sharma, K.H.S., Manohar, M.G., Kumari, R., Ahammad, S.H." Human activity recognition using deep learning", International Journal of Emerging Trends in Engineering Research 7(11), pp. 536-541.

19. Inthiyaz, S., Madhav, B.T.P., Kishore, P.V.V.” Flower image segmentation with PCA fused colored covariance and gabor texture features based level sets", Ain Shams Engineering Journal 9(4), pp. 3277-3291.

20. Inthiyaz, S., Kishore, P.V.V., Madhav, B.T.P.," Pre-informed level set for flower image segmentation" Smart Innovation, Systems and Technologies 78, pp. $11-20$

21. Inthiyaz, Syed; Madhav, B. T. P.; Kishore, P. V. V., "Flower image segmentation with PCA fused colored covariance and gabor texture features based level sets"
Ain Shams Engineering Journal (2018) 9(4) 3277-3291, 10.1016/j.asej.2017.12.007.

22. Swain, G, "Very High Capacity Image Steganography Technique Using Quotient Value Differencing and LSB Substitution" Arabian Journal For Science And Engineering, April, 2019, 10.1007/s13369-018-3372-2

23. Ahammad, S.H., Rajesh, V., Venkatesh, K.N., Nagaraju, P., Rao, P.R., Inthiyaz, S.," Liver segmentation using abdominal CT scanning to detect liver disease area", International Journal of Emerging Trends in Engineering Research 7(11), pp. 664-669. 